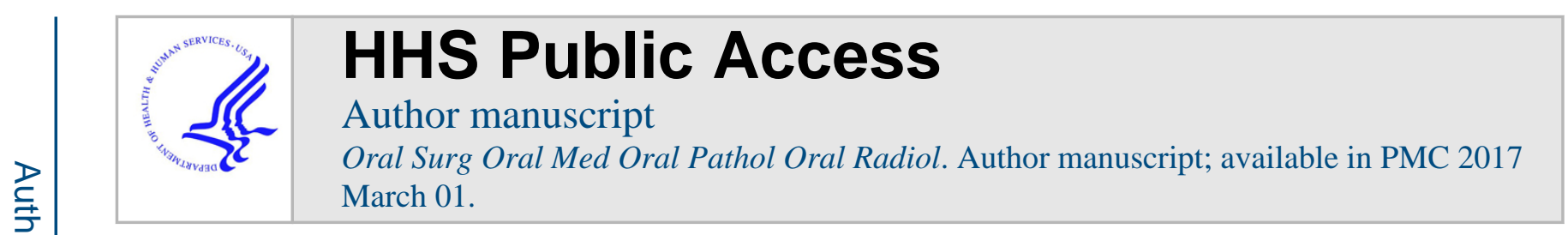

Published in final edited form as:

Oral Surg Oral Med Oral Pathol Oral Radiol. 2016 March ; 121(3): 290-300.e2. doi:10.1016/j.oooo. 2015.10.020.

\title{
A novel multimodal optical imaging system for early detection of oral cancer
}

\author{
Bilal H. Malik, Ph.D. ${ }^{1}$, Joey M. Jabbour, Ph.D. ${ }^{1}$, Shuna Cheng, Ph.D. ${ }^{1}$, Rodrigo Cuenca, B.S. \\ ${ }^{1}$, Yi-Shing Lisa Cheng, Ph.D. ${ }^{2}$, John M. Wright, DDS ${ }^{2}$, Javier A. Jo, Ph.D. ${ }^{1}$, and Kristen C. \\ Maitland, Ph.D. ${ }^{1, *}$ \\ ${ }^{1}$ Department of Biomedical Engineering, Texas A\&M University, College Station, TX 77843 \\ ${ }^{2}$ Department of Diagnostic Sciences, Texas A\&M University Health Science Center - Baylor \\ College of Dentistry, Dallas, TX 75246
}

\begin{abstract}
Objectives-Several imaging techniques have been advocated as clinical adjuncts to improve identification of suspicious oral lesions. However, these have not yet shown superior sensitivity or specificity over conventional oral examination techniques. We developed a multimodal, multiscale optical imaging system that combines macroscopic biochemical imaging of fluorescence lifetime imaging (FLIM) with subcellular morphologic imaging of reflectance confocal microscopy (RCM) for early detection of oral cancer. We tested our system on excised human oral tissues.
\end{abstract}

Study Design-A total of four tissue specimen were imaged. These specimens were diagnosed as one each: clinically normal, oral lichen planus, gingival hyperplasia, and superficially-invasive squamous cell carcinoma (SCC). The optical and fluorescence lifetime properties of each specimen were recorded.

Results-Both quantitative and qualitative differences between normal, benign and SCC lesions can be resolved with FLIM-RCM imaging. The results demonstrate that an integrated approach based on these two methods can potentially enable rapid screening and evaluation of large areas of oral epithelial tissue.

Conclusions-Early results from ongoing studies of imaging human oral cavity illustrate the synergistic combination of the two modalities. An adjunct device based on such optical characterization of oral mucosa can potentially be used to detect oral carcinogenesis in early stages.

*Corresponding Author: Kristen C. Maitland, Department of Biomedical Engineering, Texas A\&M University, 5045 Emerging Technologies Building, 3120 TAMU, College Station, TX 77843. Phone: 979-845-1864, Fax: 979-845-4450; kmaitland @ tamu.edu.

Publisher's Disclaimer: This is a PDF file of an unedited manuscript that has been accepted for publication. As a service to our customers we are providing this early version of the manuscript. The manuscript will undergo copyediting, typesetting, and review of the resulting proof before it is published in its final citable form. Please note that during the production process errors may be discovered which could affect the content, and all legal disclaimers that apply to the journal pertain.

Conflict of Interest Statement: None declared. 


\section{Introduction}

Comprehensive oral examination is the standard method to screen the oral cavity for potentially malignant disorders. Based on the clinical diagnosis through visual inspection and palpation, the clinician performs one or more biopsies, followed by histopathological diagnosis [1]. The overall clinical process is subject to interpretation and relies heavily on the experience of the clinician. Moreover, premalignant lesions are often multifocal and clinically similar to benign lesions, making it particularly difficult to select the representative site for biopsy and accurately diagnose a large clinically abnormal area [2]. Techniques that can identify oral precancers and cancers more accurately than visual inspection alone are needed to guide tissue biopsy and resection.

A number of optical imaging modalities have been proposed to noninvasively differentiate between normal, premalignant and malignant lesions within the oral cavity [3-8].

Autofluorescence imaging has emerged as one of such techniques and it has shown promise and is used clinically [9-11]. Squamous cell carcinogenesis is accompanied by complex morphologic and biochemical changes that contribute to changes in autofluorescence $[12,13]$. Some of these alterations, such as thickening of the epithelium, increase in the vascularity, and changes in the metabolic pathways, can cause changes and shifts in the native fluorescence characteristics of the tissue, which can potentially be used to discriminate malignant and/or premalignant lesions from the surrounding normal tissue [12]. For instance, restructuring of extracellular matrix in subepithelial stroma during oral carcinogenesis results in collagen degradation accompanied by loss of fluorescence and decrease in scattering, and therefore can serve as a relevant biomarker [12]. Nevertheless, while devices based on autofluorescence imaging have shown relatively high sensitivity in early detection of oral malignant lesions, the poor specificity of these devices does not support their routine use [14-17].

More advanced optical imaging techniques, including but not limited to confocal microscopy [4,18-20], nonlinear microscopy [21], optical coherence tomography [22-24] and fluorescence lifetime imaging $[25,26]$, can provide a more mechanistic basis for determining disease progression. Fluorescence lifetime imaging (FLIM) is a potentially attractive technique for detection of epithelial cancers [3,4,25-27]. It is essentially a technique to map the spatial distribution of excited state lifetimes within a sample. In most embodiments, a pulsed or modulated light source excites the sample, and the emitted fluorescence is measured in a time-resolved manner resulting directly in decay curves. The fluorescence lifetime, which is a measure of the time that a molecule remains in its excited state before returning to ground state, is extremely sensitive to the molecule's microenvironment. In addition, lifetime measurements are less sensitive to intensity artifacts and photobleaching and, thus, are more robust for clinical applications. FLIM can also exploit the tissue autofluorescence, thus obviating the need for administering exogenous contrast agents [27]. We recently demonstrated multispectral FLIM endoscopy by imaging a malignant lesion in the hamster cheek pouch model in vivo [28] and also reported a handheld probe for FLIM with the ability to access and image the human oral cavity in vivo [29]. More importantly, our implementation of FLIM offered macroscopic field of view which is critical to rapidly scan large and/or multiple lesions in the oral cavity. 
High resolution imaging techniques such as confocal microscopy provide complementary architectural information and have shown significant clinical potential as an alternative means for detection of cancers of the epithelium [18]. Confocal microscopy is essentially an optical microscopy technique with enhanced resolution and contrast by rejection of out-offocus light by a pinhole (spatial filter) in the confocal plane of the objective lens. An added benefit critical for imaging thick tissue is the "optical sectioning" principle of confocal microscopy allowing selective imaging of the focal plane and removal of signal from depths above and below the plane of interest. Specifically, reflectance confocal microscopy (RCM) collects the 'reflected' or backscattered light from the sample. In the field of tissue imaging, $\mathrm{RCM}$ is able to image tissue microstructure with subcellular resolution both in lateral and axial directions, and can detail architectural changes associated with benign, premalignant, and malignant lesions, allowing label-free detection [20]. Both ex vivo and in vivo RCM have shown the ability to image cellular morphology in real time with resolution comparable to histology [20,30]. However, in vivo application of RCM to image the oral mucosa has been somewhat limited, mainly due to the challenges associated with accessing all parts of the oral cavity.

Our group has previously demonstrated the utility of using FLIM and RCM to image oral tissue in animal models $[4,29]$. While the use of animal models can provide the necessary complexity to validate the engineering design, the tissue structure and biochemistry are not equivalent to human tissue. Here, we have applied a modified multimodal optical imaging setup which allows the imaging of human oral tissue ex vivo. Specifically, FLIM is used to acquire relatively large scale multispectral images of oral tissue biopsies ex vivo with a single image scan. This is followed by RCM to identify and characterize tissue morphology at a microscopic level. The study was designed to demonstrate the dual modality system's ability towards evaluation of normal, benign, premalignant and malignant human oral lesions, and is focused on both qualitative and quantitative characterization of lesions, the comparison of tissue morphology and biochemical composition, and illustrating the synergy of the combined FLIM-RCM system. This study represents a transition from testing this novel system in the animal model to testing it on excised human normal or pathological tissue, a procedure we believe is a necessary step towards future clinical trial and the ultimate clinical application for early detection of oral cancer.

\section{Materials and Methods}

\section{Oral tissue collection}

The tissue collection and imaging protocols were approved by the Institutional Review Boards at Texas A\&M University (TAMU) and TAMU-Baylor College of Dentistry. Written informed consent was obtained from all patients who either had osseous surgery or crown lengthening procedures for removal of clinically normal excess tissue or had clinically benign, premalignant or malignant lesions that required biopsies. After surgical excision, the tissue samples were immediately placed in phosphate buffered saline solution and kept on ice in order to minimize tissue degradation. Both FLIM and RCM imaging were performed within 30 minutes following tissue collection. 


\section{FLIM imaging}

The tissue samples were placed in a quartz petri dish and then positioned under the objective lens of the FLIM module. The detailed configuration of this system and the ensuing analysis is described in detail elsewhere and in the accompanying supplementary information (Supplementary Figure 1) [29]. Time-resolved fluorescence data was collected sequentially with a photomultiplier-tube (PMT) detector coupled to a high-speed digitizer. Finally, nine images were generated from each FLIM scan: absolute integrated fluorescence intensity, normalized integrated fluorescence intensity and average fluorescence lifetime maps corresponding to each of the three emission channels.

\section{RCM imaging}

Immediately following FLIM acquisition, the tissue samples were treated with 5\% acetic acid for $\sim 60$ seconds to increase the amount of reflected light from cells at or near the tissue surface. The current embodiment of our RCM module has been previously described in detail and in the supplementary information [31]. The illumination light was scanned on the sample in a rectangular pattern and coupled to the microscope objective through an image relay. The backscattered light was focused onto a pinhole for confocal detection, followed by a PMT detector. Finally, a digitizer was used for signal collection and real-time image formation.

\section{Histopathology}

Following imaging, biopsies were placed in $10 \%$ buffered formalin and processed for hematoxylin and eosin (H\&E) histology. The stained sections were reviewed by the study pathologists (YLC and JMW) who were blinded to the results of optical imaging. The sections were assigned to one of the following categories: normal, benign (lichen planus), mild dysplasia, moderate dysplasia, severe dysplasia, or cancer (squamous cell carcinoma). If any of the sections were found to have more than one disease state, then the most severe diagnosis was assigned to that biopsy. A total of $n=4$ tissue biopsies were imaged using either or both the imaging modalities. These specimen were diagnosed as one each: clinically normal, oral lichen planus, gingival hyperplasia, and superficially invasive squamous cell carcinoma.

\section{Results}

\section{FLIM can visualize the biochemical makeup of the oral tissue}

The left panel of images in Figure 1 shows the representative FLIM maps and corresponding histology image from a clinically normal oral tissue. The specific tissue sample was acquired from a crown lengthening procedure wherein excess gingival tissue was surgically removed for future tooth restoration. As indicated, the images correspond to normalized fluorescence intensity and average fluorescence lifetime values, corresponding to the three emission wavelength bands (left to right). These three spectral bands cover the range of emissions of biologically most relevant endogenous fluorophores. The most important sources of autofluorescence in this range are collagen (peak emission: $390 \mathrm{~nm}$, channel 1), NADH (peak emission: $450 \mathrm{~nm}$, channel 2) and FAD (peak emission: $550 \mathrm{~nm}$, channel 3). 
Although tissue autofluorescence usually exhibits complex fluorescence decay profiles, average lifetime can still provide useful contrast between different types and/or pathological states of the tissue. Across the area of the tissue section, the corresponding normalized intensity and lifetime images were relatively uniform, as depicted in Figure 1. The normalized intensity images yielded mean values of $0.27 \pm 0.08,0.51 \pm 0.05$, and $0.23 \pm$ 0.04 across the tissue surface, for channels 1, 2 and 3, respectively. Although, the peak emission wavelength of collagen is usually below $400 \mathrm{~nm}$, the relatively long 'red-tail' of the emission profile results in a significant contribution to the first channel, and to some extent, the second channel [32]. For this reason, the corresponding lifetime values, calculated as $4.84 \pm 0.43,3.95 \pm 0.38$ and $3.79 \pm 0.28 \mathrm{~ns}$, respectively, resemble those of collagen which is the predominant fluorophore in most tissue types. Nevertheless, the contribution of NADH manifests as a decrease in average lifetime in the second channel relative to the first channel. The third channel represents fluorescence mainly from FAD, although depending on the pathology of the tissue, other factors, such as porphyrin, can play a role as well [5]. Note that the left border of the tissue had a region where the underlying connective tissue was exposed, resulting in a slight increase in the contribution of collagen in that area.

The right panel of images in Figure 1 shows FLIM maps of a tissue biopsy representative of oral lichen planus (OLP). OLP is a benign inflammatory condition of unknown etiology but with immune mediated pathogenesis. The intensity map in channel 1 shows an area of relatively high signal towards the upper right region of the biopsy. This area was found to be shy of epithelium during histopathological evaluation, which resulted in a significant relative increase in the signal from collagen in the connective tissue. This contrast can also be seen in the fluorescence lifetime maps (Figure 1k-m) where the same region exhibits longer lifetime values in comparison to the rest of the tissue section. While in channels 1 and 2 , this is mainly because of collagen in the lamina propria, longer lifetime values in channel 3 may also be attributed to relatively higher concentration of porphyrins present in the blood vessels within the connective tissue.

\section{FLIM can resolve differences between precancerous and cancerous regions of a biopsy}

The top panel in Figure 2 shows FLIM images from a tissue biopsy which was acquired from the right lateral region of the tongue. The biopsy was diagnosed as superficially invasive squamous cell carcinoma. While most of the surface was covered by stratified squamous epithelium showing severe epithelial dysplasia (Figure $2 \mathrm{~g}$ ), a few tumor islands of basaloid cells exhibiting abnormal mitosis and hyperchromatism could be observed in the lamina propria above the skeletal muscle (Figure 2h). Although most of the FLIM features do not display any significant variation across the surface of the tissue and are relatively uniform, the lifetime map of channel 3 exhibits a marked increase in the upper left quadrant of the map. Location specific histological evaluation showed that region to have the invasive tumor islands while the rest of the tissue region was classified as moderate to severe dysplasia. The mean average lifetime value in the third channel across the tumor region was calculated to be $5.2 \pm 0.37 \mathrm{~ns}$ in comparison to slightly lower value of $4.1 \pm 0.44 \mathrm{~ns}$ for the rest of the tissue section, showing the ability of FLIM to potentially differentiate between 
premalignant and malignant lesions. RCM images were acquired from the center of this biopsy, with the location indicated by a box in Figure 2e; the results are discussed below.

Figure 3 provides a series of histograms, along with the corresponding bar plots, comparing the corresponding FLIM features of the aforementioned tissue sections: clinically normal, benign (OLP) and premalignant (dysplasia) lesions. Specifically, Figures 3a-c compare the distribution of normalized intensity maps to illustrate the changes in relative contributions to overall fluorescence from the three emission bands. Collagen is the largest contributor to total fluorescence generated in the superficial oral tissue and hence almost all of signal in Channel 1 and a significant portion of signal in Channel 2 can be attributed to collagen fluorescence. It is expected that the intensity of collagen fluorescence would decrease with premalignant and malignant transformation, due to collagen degradation and thickening of epithelium [12]. While Figure 3d does not show a statistically significant overall decrease in the respective signals for benign and premalignant tissue, the normal tissue did exhibit slightly higher average fluorescence intensity in comparison to other tissue types in Channels 1 and 2. Note that the benign lesion shows (Figures 3a and 3d) least amount of contribution to overall fluorescence in Channel 1 . This behavior can possibly be due to a marked increase in the penetration of lymphocytes in the lamina propria as can be seen in the corresponding histology section in Figure 1n. The cells of inflammatory infiltrate are known to cause collagen degradation [33]. Only the information in channel 3 exhibited a significant difference for both benign and premalignant lesions in comparison to normal tissue; however, the benign lesion displayed a much wider distribution in comparison to both normal and premalignant tissue sections.

Figures 3e-h show and compare the distribution of fluorescence lifetime maps of the three pathologically different lesions. The lifetime in channel 1 showed a significant decrease in overall distribution in the case of benign and premalignant lesions in comparison to normal tissue, which, again, may be attributed to degradation of collagen in the lamina propria. Channel 2 showed a slight but not significant decrease in the lifetime for benign and premalignant lesions. Similar observation has been made by Skala et al., wherein a decrease was observed in the lifetime and contribution of NADH in precancerous and cancerous lesions in comparison to normal tissue [26]. The lifetime in channel 3 showed a slight increase in the case of premalignancy in comparison to both benign and normal tissue sections. While such an increase, attributed to change in lifetime of FAD, has previously been observed in literature, it can also be a manifestation of porphyrin (lifetime $>6 \mathrm{~ns}$ ). Porphyrin's relative presence and contribution has been known to increase with malignant transformation $[26,34,35]$.

\section{RCM can detail architectural features of the oral epithelium}

In comparison to FLIM's ability to provide biochemical and molecular contrast, RCM probes the tissue architecture and microanatomy, and can generate images which can be directly correlated to the conventional histological evaluation of the specimen. Figure 4 shows a comparison of a transverse histological image and the corresponding en face confocal images of a clinically normal gingiva tissue. The confocal images were obtained at different depths within the epithelium of the tissue. Bright nuclei and cell borders on a 
background of relatively darker cytoplasm can be observed throughout the thickness of the epithelium. The images from the middle of the epithelium to the basal layer show a noticeable increase in the cell density and nuclear-to-cytoplasmic (N/C) ratio - features which compare well with the corresponding regions of the histology section. Such a progression in the pattern of cells is typical of normal oral epithelial tissue.

Figure 5 shows another set of RCM images taken from a tissue biopsy suspected of inflammation and which was later classified as gingival hyperplasia. In comparison to the images in Figure 4, there is a marked increase in the density of cells, indicative of hyperplasic growth of the tissue. The peg like structures of rete ridges can also be observed in the images at the interface of the epithelium and lamina propria, towards the upper right quadrant in Figure 5e. Note that while we were unable to image any ulcerated regions due to their peripheral locations on the tissues, RCM has the ability to differentiate between the epithelial cells and the stroma [20].

\section{Differences in morphologic features between normal, benign and precancerous tissues can be quantified using RCM-We next assessed whether} the images generated by RCM could be used to identify the presence of oral premalignancy. Figure 2i-k depicts images from the superficial invasive carcinoma tissue imaged by FLIM, from the region marker in Figure 2e. This area was histopathologically interpreted as severe dysplasia. In comparison to normal and benign tissue sections, this confocal image mosaic showed an increase in the N/C ratio at the respective depths within the epithelial tissue, and a much smaller change across the depth of the epithelium.

These differences were quantified by segmenting the images to isolate the cell nuclei. We utilized a custom-written software routine programmed specifically for segmentation of RCM images of the oral epithelium [36]. The percentage of change in N/C ratio across the thickness of the epithelium was found to be higher for the both clinically normal tissue and the benign (hyperplasia) lesion in comparison to dysplasia. Specifically, the change in N/C ratio across middle to bottom of tissue was measured to be $\sim 71 \%$ and $\sim 58 \%$ for normal and benign tissue, respectively. In contrast, this change was less than $3 \%$ in the case of dysplastic tissue. This is typical of stratified squamous epithelium wherein normal tissue exhibits marked change in N/C ratio across the depth of the tissue as the basal cells divide and mature, and move upward within the epithelium. This difference becomes less noticeable with the progression of premalignancy as atypical nuclei tend to move up in the epithelium.

\section{Discussion}

In this study, FLIM and RCM imaging combine metabolic and compositional mapping with morphological information to characterize different stages of oral cancer development. The ability to realize clinical potential of this integrated approach is underscored by the complimentary nature of information provided by these modalities. While some of the FLIM features of clinically normal and premalignant tissue showed significant differences, the comparisons between normal and benign, or benign and premalignant tissue sections were mostly insignificant. However, comparison of RCM images of normal, benign and 
premalignant tissue sections showed distinctive differences in percentage change of N/C ratio. We believe that the synergistic combination of macroscopic FLIM (high sensitivity, low specificity) to rapidly scan a large area of a biopsy, or potentially oral cavity in vivo, and microscopic RCM (high sensitivity, high specificity) to provide depth-sensitive histologylike architectural information can overcome the limitations of individual imaging techniques, and provide a practical and objective adjunct to conventional oral screening methods.

Our results from FLIM imaging also demonstrate that the additional dimension of fluorescence lifetime may enhance the ability of clinicians to distinguish premalignancy from malignancy. As seen in Figure 2, the lifetime map in the third channel exhibits most significant contrast between the areas identified as dysplasia and carcinoma. The increase in the fluorescence lifetime may be attributed to the elevated levels of porphyrin in the cancerous area. During the process of malignant transformation, porphyrin level in tissue can increase due to alterations in both heme biosynthetic and amino acid degradation pathways [37]. Note that while such an increase can potentially serve as a marker of malignant transformation, concentration of porphyrins in blood is much higher in comparison. Therefore, areas of tissue which are shy of epithelium (such as ulcerated regions) and where connective tissue and the blood vessels therein are relatively exposed can confound the interpretation. This can be a potential limitation of FLIM in discriminating malignant lesions from benign regions of the tissue.

The architectural details in RCM images can be quantified to more objective parameters (N/C ratio and size of nuclei) which can be directly correlated to that seen in histology. Thickness of the oral epithelium is another feature which can be used to characterize precancerous and cancerous regions within the tissue; however, individuals across different ages, genders, and races can exhibit significant variations in the size of the epithelial thickness [38]. In addition, the thickness of the layer also varies substantially across different regions of the mouth. For instance, the epithelial layer has been measured to be 99 $+/-22 \mu \mathrm{m}$ thick at the anterior floor of the mouth in comparison to thickness of $294+/-68$ $\mu \mathrm{m}$ at buccal mucosa [39].

As mentioned earlier, the premalignant and malignant transformations within the epithelium are marked with increased cellular polarity and consequent loss of cell maturation and stratification [40]. In principal, our RCM system should be able to resolve such abnormal variations which could potentially serve as additional markers to distinguish precancerous and cancerous lesions. However, the high and disordered concentration of the nuclei can also result in decreased scattering of light and, therefore, contrast, as seen in confocal images of Figure 2 in comparison to Figure 4-5, making it difficult to identify more subtle changes at the cytological level. Our image segmentation program also calculated the mean background intensity around the nuclei in the images, and the results showed a $\sim 40 \%$ increase in the background indicating reduced contrast for diseased tissue. This is also in agreement with predictions of light scattering theory for closely spaced structures, which indicates that such geometry and distance between nuclei would result in destructive interference of the backscattered light [41]. 
One of the limitations of RCM imaging is the penetration depth which restricts how deep we can image within the biological tissue. In both our experience and reports by other groups, RCM images have been acquired at $>300 \mu \mathrm{m}$ in the oral epithelium [42]. While this penetration depth is relatively low in comparison to other imaging modalities such as optical coherence tomography and multi-photon microscopy, most normal values of epithelial thickness within the oral cavity have been noted to be less than $300 \mu \mathrm{m}$ [39]. More importantly, the areas of the oral cavity most commonly identified with oral cancer are lateral tongue ( $200 \mu \mathrm{m}$ average thickness) and anterior floor of the mouth $(\sim 100 \mu \mathrm{m}$ average thickness), both of which are well within the range of RCM. Nevertheless, the epithelium can show significant increase in thickness during progression of precancer, and potentially limit our ability to image through to the basal cell layer. In addition, regions with severe hyperkeratosis can also reduce the penetration of light due to increased backscattering by the keratin layer. In such instances, the sensitivity of FLIM to the metabolic state could become increasingly critical in its ability to detect and distinguish the lesion(s); however, hyperkeratosis can also confound the overall fluorescence signal due to similarities in emission of keratin and collagen.

Recently, we have modified the system for use in vivo in the oral cavity of patients. Our preliminary results are promising, demonstrating that this data presented here, acquired $e x$ vivo, is translatable to in vivo imaging. In vivo FLIM imaging of intact tissue is less susceptible to image variation due to exposure of connective tissue in positioning of biopsies, as seen in Figure 1. In vivo RCM imaging is more susceptible to motion artifacts because of the high resolution imaging; however, the 7 frames per second frame rate is sufficient for image capture. While presence of blood in vivo can limit the penetration of light in tissue, this problem is circumvented in the case of oral mucosa wherein the blood vessels are predominantly present in the connective tissue underneath the epithelial layer. Since both the imaging modalities primarily probe the epithelium, the effect of circulating blood on our ability to image the tissue is not significant. A potential limitation with RCM is the relatively small field of view. Image mosaicking may be applied in the future to expand the area of the high resolution imaging region. We are in the process of building a database of FLIM and RCM images of a variety of pathologies including benign, premalignant and malignant conditions. This database will be used to perform multivariate statistical analysis in order to identify the most important imaging parameters which best differentiate between pathologies along the transformation path of squamous cell carcinoma.

\section{Supplementary Material}

Refer to Web version on PubMed Central for supplementary material.

\section{Acknowledgments}

Funding Agency: This work was supported by the National Institutes of Health (R01 CA138653). 


\section{References}

1. Rethman MP, Carpenter W, Cohen EE, et al. Evidence-based clinical recommendations regarding screening for oral squamous cell carcinomas. J Am Dent Assoc. May; 2010 141(5):509-20. [PubMed: 20436098]

2. Lingen MW, Kalmar JR, Karrison T, Speight PM. Critical evaluation of diagnostic aids for the detection of oral cancer. Oral Oncol. Jan; 2008 44(1):10-22. [PubMed: 17825602]

3. Fatakdawala H, Poti S, Zhou F, et al. Multimodal in vivo imaging of oral cancer using fluorescence lifetime, photoacoustic and ultrasound techniques. Biomed Opt Express. 2013; 4(9):1724-41. [PubMed: 24049693]

4. Jabbour JM, Cheng S, Malik BH, et al. Fluorescence lifetime imaging and reflectance confocal microscopy for multiscale imaging of oral precancer. J Biomed Opt. Apr.2013 18(4):046012. [PubMed: 23595826]

5. Sun Y, Xie H, Liu J, et al. In vivo validation of a bimodal technique combining time-resolved fluorescence spectroscopy and ultrasonic backscatter microscopy for diagnosis of oral carcinoma. $\mathrm{J}$ Biomed Opt. 2012; 17(11):116003-03. [PubMed: 23117798]

6. Muldoon TJ, Roblyer D, Williams MD, Stepanek VMT, Richards-Kortum R, Gillenwater AM. Noninvasive imaging of oral neoplasia with a high-resolution fiber-optic microendoscope. Head Neck. Mar; 2012 34(3):305-12. [PubMed: 21413101]

7. Pierce MC, Schwarz RA, Bhattar VS, et al. Accuracy of in vivo multimodal optical imaging for detection of oral neoplasia. Cancer Prev Res. Jun; 2012 5(6):801-9.

8. Ahn YC, Chung JR, Wilder-Smith P, Chen ZP. Multimodality approach to optical early detection and mapping of oral neoplasia. J Biomed Opt. Jul.2011 16(7)

9. Awan KH, Morgan PR, Warnakulasuriya S. Evaluation of an autofluorescence based imaging system (VELscope (TM)) in the detection of oral potentially malignant disorders and benign keratoses. Oral Oncol. Apr; 2011 47(4):274-77. [PubMed: 21396880]

10. Pierce MC, Schwarz RA, Rosbach K, et al. Wide-field and high-resolution optical imaging for early detection of oral neoplasia. Proc. SPIE. 2010:7548.

11. Roblyer D, Kurachi C, Stepanek V, et al. Objective Detection and Delineation of Oral Neoplasia Using Autofluorescence Imaging. Cancer Prev Res. May; 2009 2(5):423-31.

12. Pavlova I, Williams M, El-Naggar A, Richards-Kortum R, Gillenwater A. Understanding the biological basis of autofluorescence imaging for oral cancer detection: High-resolution fluorescence microscopy in viable tissue. Clin Cancer Res. Apr 15; 2008 14(8):2396-404. [PubMed: 18413830]

13. Pavlova I, Sokolov K, Drezek R, Malpica A, Follen M, Richards-Kortum R. Microanatomical and biochemical origins of normal and precancerous cervical autofluorescence using laser-scanning fluorescence confocal microscopy. Photochem Photobiol. May; 2003 77(5):550-5. [PubMed: 12812299]

14. McNamara KK, Martin BD, Evans EW, Kalmar JR. The role of direct visual fluorescent examination (VELscope) in routine screening for potentially malignant oral mucosal lesions. Oral Surg Oral Med Oral Pathol Oral Radiol. Nov; 2012 114(5):636-43. [PubMed: 23083477]

15. Hanken H, Kraatz J, Smeets R, et al. The detection of oral pre- malignant lesions with an autofluorescence based imaging system (VELscope) - a single blinded clinical evaluation. Head Face Med. 2013; 9:23. [PubMed: 23967796]

16. Scheer M, Neugebauer J, Derman A, Fuss J, Drebber U, Zoeller JE. Autofluorescence imaging of potentially malignant mucosa lesions. Oral Surg Oral Med Oral Pathol Oral Radiol Endod. May; 2011 111(5):568-77. [PubMed: 21429774]

17. Rana M, Zapf A, Kuehle M, Gellrich NC, Eckardt AM. Clinical evaluation of an autofluorescence diagnostic device for oral cancer detection: a prospective randomized diagnostic study. Eur J Cancer Prev. Sep; 2012 21(5):460-6. [PubMed: 22217551]

18. Jabbour JM, Saldua MA, Bixler JN, Maitland KC. Confocal Endomicroscopy: Instrumentation and Medical Applications. Ann Biomed Eng. Oct 13.2011 
19. Maitland KC, Gillenwater AM, Williams MD, El-Naggar AK, Descour MR, Richards-Kortum RR. In vivo imaging of oral neoplasia using a miniaturized fiber optic confocal reflectance microscope. Oral Oncol. Nov; 2008 44(11):1059-66. [PubMed: 18396445]

20. Clark AL, Gillenwater AM, Collier TG, Alizadeh-Naderi R, El-Naggar AK, Richards-Kortum RR. Confocal microscopy for real-time detection of oral cavity neoplasia. Clin Cancer Res. Oct 15; 2003 9(13):4714-21. [PubMed: 14581341]

21. Skala MC, Squirrell JM, Vrotsos KM, et al. Multiphoton microscopy of endogenous fluorescence differentiates normal, precancerous, and cancerous squamous epithelial tissues. Cancer Res. Feb 15; 2005 65(4):1180-86. [PubMed: 15735001]

22. Wilder-Smith P, Lee K, Guo SG, et al. In Vivo Diagnosis of Oral Dysplasia and Malignancy Using Optical Coherence Tomography: Preliminary Studies in 50 Patients. Lasers Surg Med. Jul; 2009 41(5):353-57. [PubMed: 19533765]

23. Smith LE, Hearnden V, Lu ZH, et al. Evaluating the use of optical coherence tomography for the detection of epithelial cancers in vitro. J Biomed Opt. Nov.2011 16(11)

24. Clark AL, Gillenwater A, Alizadeh-Naderi R, El-Naggar AK, Richards-Kortum R. Detection and diagnosis of oral neoplasia with an optical coherence microscope. J Biomed Opt. Nov-Dec;2004 9(6):1271-80. [PubMed: 15568948]

25. Shrestha S, Park J, Pande P, Applegate BE, Jo JA. Multimodal Optical Imaging for Simultaneous In-Vivo Morphological and Biochemical Characterization of Oral Epithelial Cancer. Conf Proc IEEE Eng Med Biol Soc. 2010:1970-73. [PubMed: 21096785]

26. Skala MC, Riching KM, Gendron-Fitzpatrick A, et al. In vivo multiphoton microscopy of NADH and FAD redox states, fluorescence lifetimes, and cellular morphology in precancerous epithelia. Proc Natl Acad Sci U S A. Dec 4; 2007 104(49):19494-99. [PubMed: 18042710]

27. Sun Y, Phipps J, Elson DS, et al. Fluorescence lifetime imaging microscopy: in vivo application to diagnosis of oral carcinoma. Opt Lett. Jul 1; 2009 34(13):2081-3. [PubMed: 19572006]

28. Cheng S, Rico-Jimenez JJ, Jabbour J, et al. Flexible endoscope for continuous in vivo multispectral fluorescence lifetime imaging. Opt Lett. May 1; 2013 38(9):1515-7. [PubMed: 23632536]

29. Cheng SN, Cuenca RM, Liu BA, et al. Handheld multispectral fluorescence lifetime imaging system for in vivo applications. Biomed Opt Express. Mar 1; 2014 5(3):921-31. [PubMed: 24688824]

30. Rajadhyaksha M, Anderson RR, Webb RH. Video-rate confocal scanning laser microscope for imaging human tissues in vivo. Applied Opt. Apr 1; 1999 38(10):2105-15.

31. Jabbour JM, Malik BH, Olsovsky C, et al. Optical axial scanning in confocal microscopy using an electrically tunable lens. Biomed Opt Express. Feb 1; 2014 5(2):645-52. [PubMed: 24575357]

32. Pu Y, Wang W, Yang Y, Alfano RR. Native fluorescence spectra of human cancerous and normal breast tissues analyzed with non-negative constraint methods. Applied Opt. 2013; 52(6):1293-301. 2013/02/20.

33. Tarpey SG, White FH. Ultrastructural morphometry of collagen from lamina propria during experimental oral carcinogenesis and chronic inflammation. J Cancer Res Clin Oncol. 1984; 107(3):183-94. [PubMed: 6429150]

34. Chen H-M, Chiang C-P, You C, Hsiao T-C, Wang C-Y. Time-resolved autofluorescence spectroscopy for classifying normal and premalignant oral tissues. Lasers Surg Med. 2005; 37(1): 37-45. [PubMed: 15954122]

35. Yuvaraj M, Udayakumar K, Jayanth V, et al. Fluorescence spectroscopic characterization of salivary metabolites of oral cancer patients. J Photochem Photobiol B. Jan 5.2014 130:153-60. [PubMed: 24333763]

36. Harris MA, Van AN, Malik BH, Jabbour JM, Maitland KC. A pulse coupled neural network segmentation algorithm for reflectance confocal images of epithelial tissue. PLoS One. 2015; 10(3):e0122368. [PubMed: 25816131]

37. Marcu, L.; French, PMW.; Elson, DS. Fluorescence lifetime spectroscopy and imaging: principles and applications in biomedical diagnostics. CRC Press; Boca Raton, Florida: 2014.

38. Vandana KL, Savitha B. Thickness of gingiva in association with age, gender and dental arch location. J Clin Periodontol. Jul; 2005 32(7):828-30. [PubMed: 15966893] 
39. Prestin S, Rothschild SI, Betz CS, Kraft M. Measurement of epithelial thickness within the oral cavity using optical coherence tomography. Head Neck. Dec; 2012 34(12):1777-81. [PubMed: 22318761]

40. Martin-Belmonte F, Perez-Moreno M. Epithelial cell polarity, stem cells and cancer. Nat Rev Cancer. Jan; 2012 12(1):23-38. [PubMed: 22169974]

41. Ishimaru, A. Wave propagation and scattering in random media. Wiley-IEEE Press; New York: 1997.

42. Meglinski IV, Bashkatov AN, Genina EA, Churmakov DY, Tuchin VV. Study of the possibility of increasing the probing depth by the method of reflection confocal microscopy upon immersion clearing of near-surface human skin layers. Quantum Electronics. Oct; 2002 32(10):875-82. 


\section{Statement of Clinical Relevance}

An imaging device for optical characterization of oral mucosa using a dual modality system based on fluorescence lifetime imaging and reflectance confocal microscopy can potentially be used to detect oral carcinogenesis in early stages. 

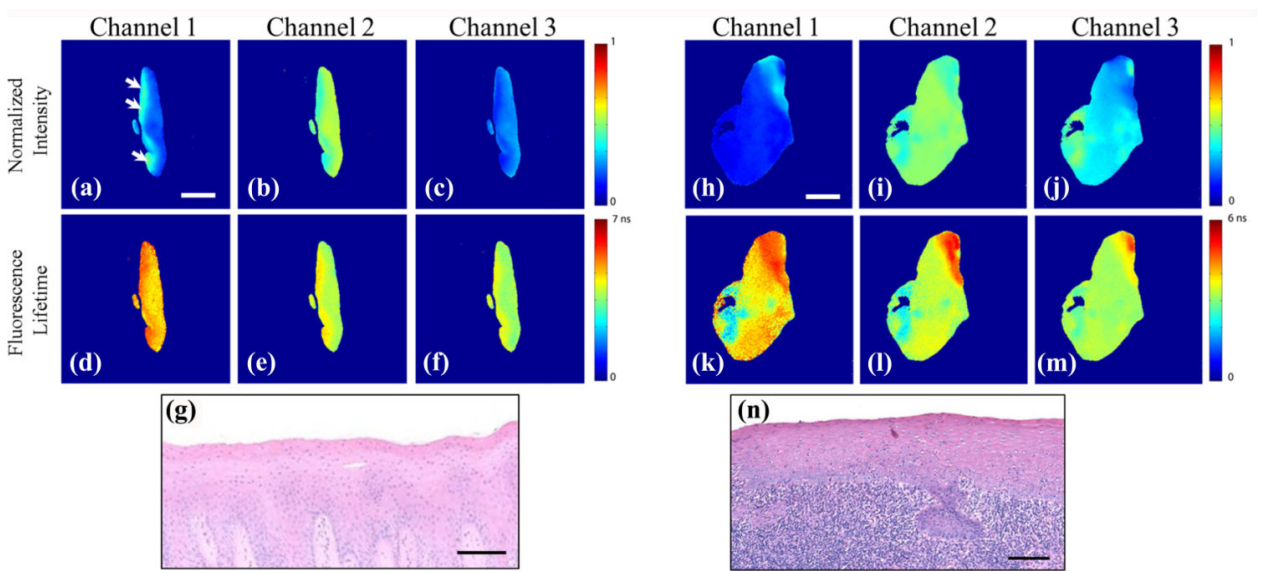

Figure 1.

(a-f) FLIM images of the clinically normal tissue section from gingiva. (a-c) normalized fluorescence intensity, and (d-f) fluorescence lifetime maps in the three channels corresponding to the emission wavelength bands of $390 \pm 20 \mathrm{~nm}$ (channel 1), $452 \pm 22.5 \mathrm{~nm}$ (channel 2) and > $500 \mathrm{~nm}$ (channel 3); (g) image of the corresponding histology section. Note that the left border of the tissue (marked by arrows) had an area where the underlying connective tissue was exposed and hence the contribution of collagen was slightly increased in that region. (h-m) FLIM images of the benign lesion classified as oral lichen planus. (h-j) normalized fluorescence intensity, and (k-m) fluorescence lifetime maps corresponding to the peak emission wavelength bands of $390 \pm 20 \mathrm{~nm}$ (channel 1), $452 \pm 22.5 \mathrm{~nm}$ (channel 2) and > $500 \mathrm{~nm}$ (channel 3); (n) image of the corresponding histology section. Scale bars: 2 $\mathrm{mm}$ in (a-f) and (h-m), $100 \mu \mathrm{m}$ in (g) and (n). 

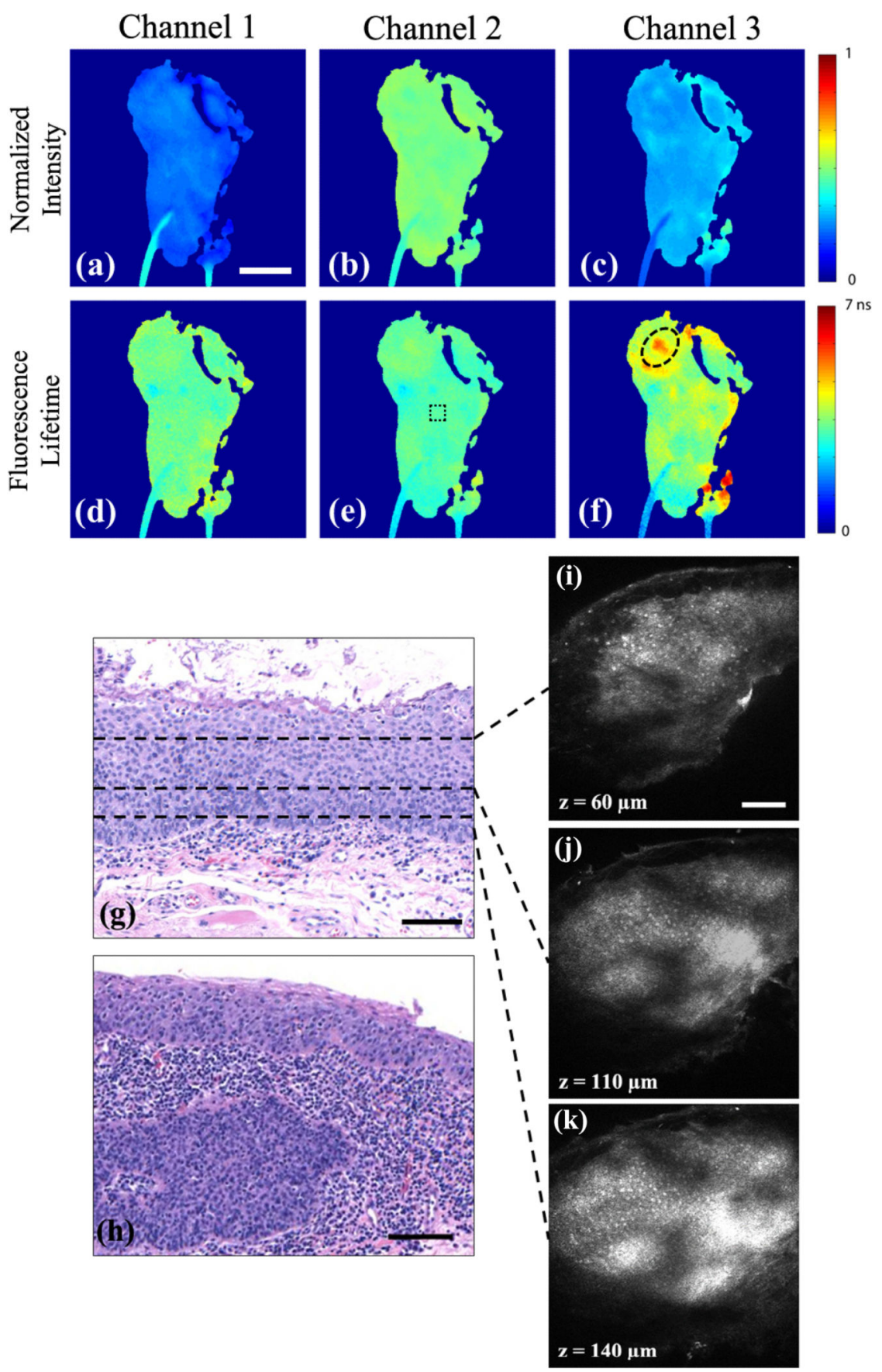

Figure 2.

FLIM and RCM images of the squamous cell carcinoma lesion. (a-c) normalized fluorescence intensity, and (d-f) fluorescence lifetime maps corresponding to the peak emission wavelength bands of $390 \pm 20 \mathrm{~nm}$ (channel 1), $452 \pm 22.5 \mathrm{~nm}$ (channel 2) and > $500 \mathrm{~nm}$ (channel 3); (g-h) histology sections corresponding to regions of dysplasia and SCC, respectively; (i-k) RCM images of the dysplastic region marked in (e). Note that both histology and RCM images show relatively unchanged N/C ratio across the depth of the epithelium. The area enclosed by dashed line in (f) depicts the region histopathologically 
classified as superficially invasive carcinoma shown in (h), while rest of the biopsy displayed severe dysplasia as depicted in (g). Scale bars: $2 \mathrm{~mm}$ in (a-f), $100 \mu \mathrm{m}$ in (g-k). The FLIM images shown here have previously been published in reference [29] and are reproduced here with permission of Optical Society of America. 
Normalized Intensity
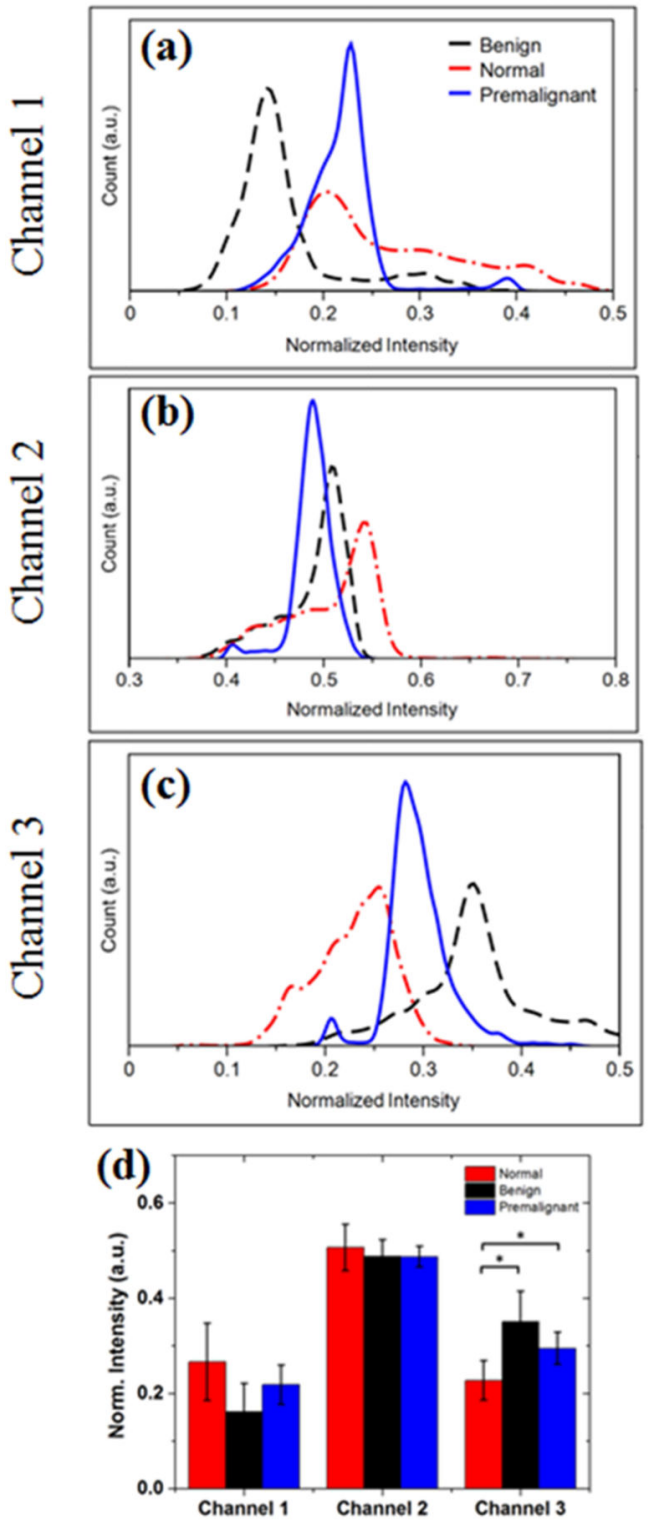

Fluorescence Lifetime
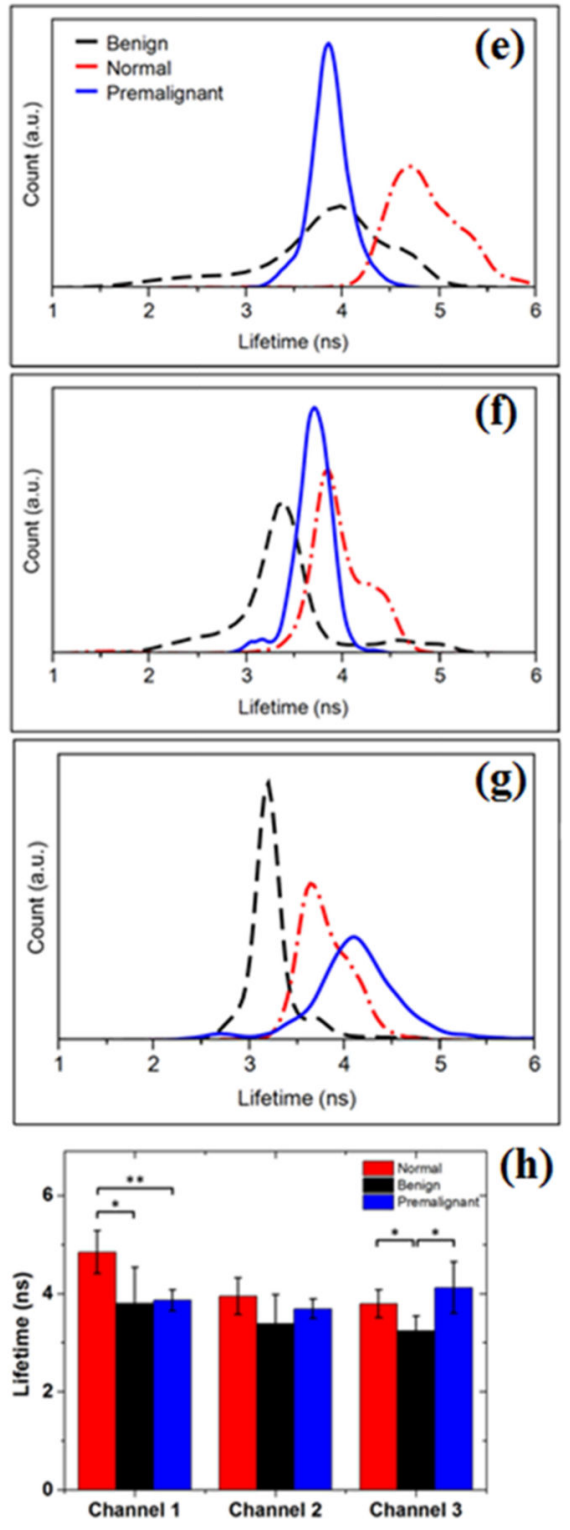

(h)

Figure 3.

Histograms of normalized fluorescence intensity images (left column) and fluorescence lifetime (right column) corresponding to channel 1 (a, e), channel 2 (b, f) and channel 3 (c, g). (d) and (h) show a comparison of the mean values of intensity and lifetime. The horizontal brackets above the bars indicate significant difference among pairs using the nonparametric Mann-Whitney U test (single asterisk, $\mathrm{p}<0.01$; double asterisks, $\mathrm{p}<0.001$ ). 


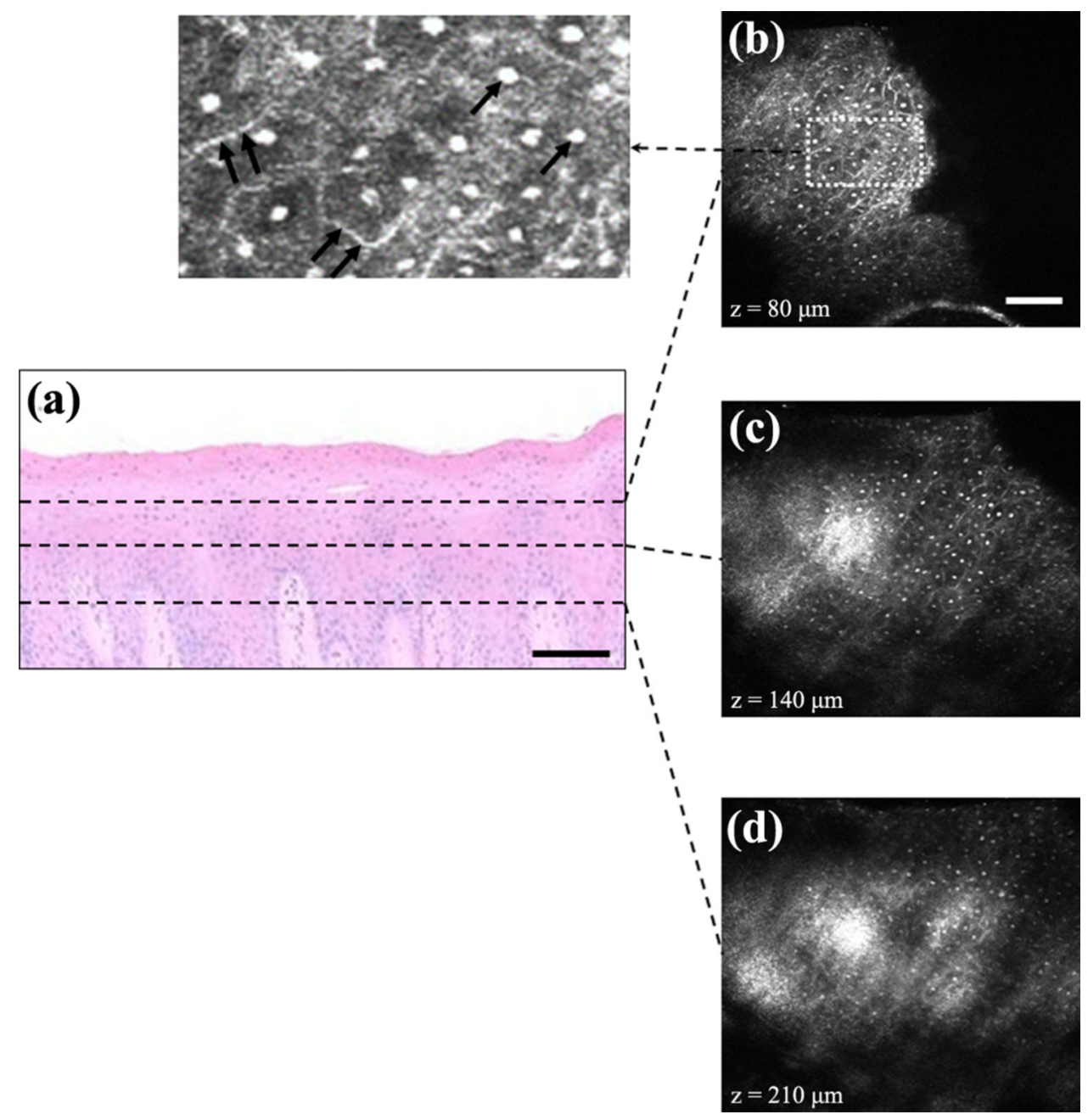

Figure 4.

Comparison of a clinically normal histology section (a) from gingiva with the corresponding en face RCM images (b-d) obtained at different depths beneath the epithelial surface. The change in N/C ratio through the thickness of the epithelium, (b) to (d), can be observed as the imaging depth increased beneath the tissue surface. Zoom in view of the boxed region in (b) shows that the nuclei (marked by arrows) and cell membranes (marked by double arrows) can clearly be identified by RCM. Scale bars: $100 \mu \mathrm{m}$. 

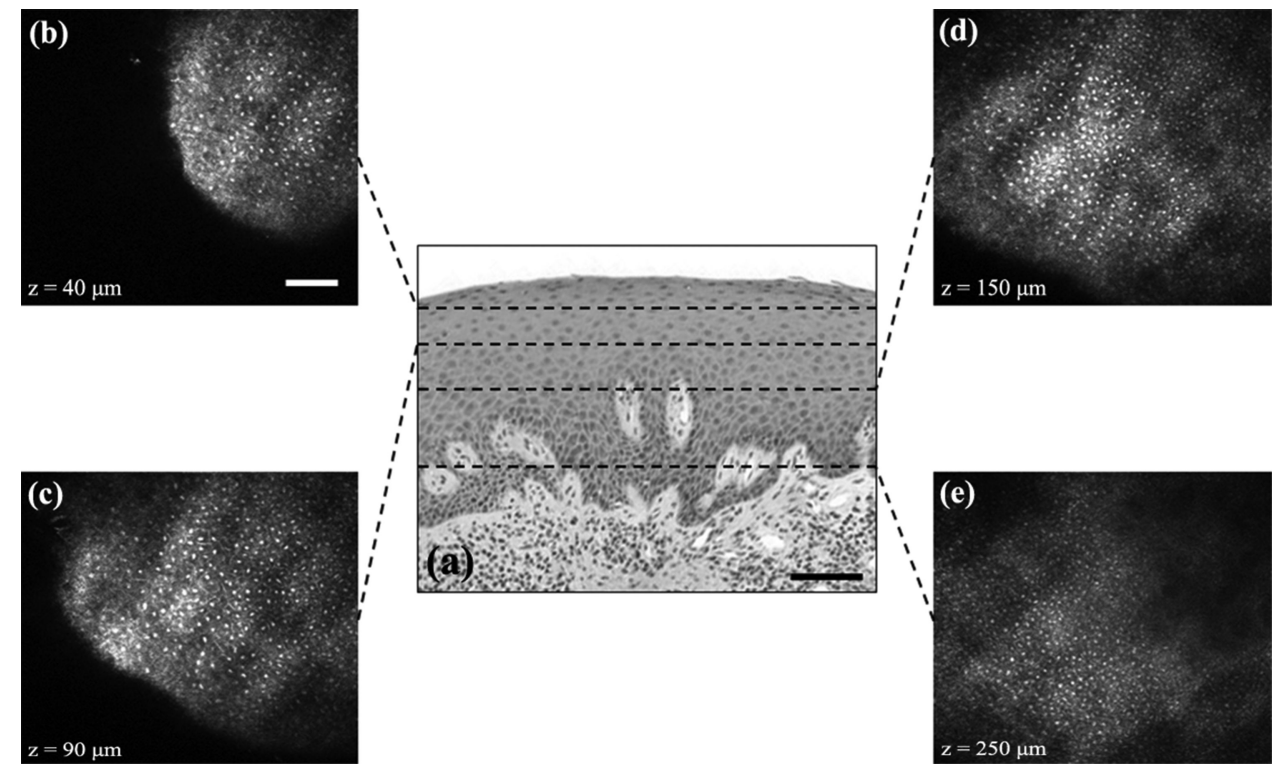

\section{(e)}

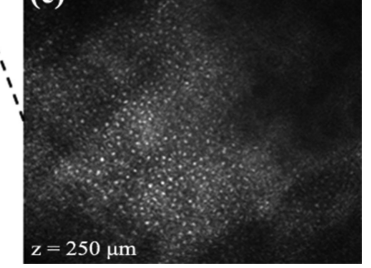

\section{Figure 5.}

Histological section (a) and RCM images (b-e) of a tissue section from gingiva region. The section was histopathologically categorized as a hyperplastic lesion that occurs in response to chronic inflammation/irritation. In comparison to clinically normal tissue, the tissue exhibits marked proliferation of cells, as seen both in histology and RCM images. Note that the variation in the field of view across the RCM images is due to the placement of tissue surface at an angle with respect to the microscope objective. The change in N/C ratio can also be noticed in (c) and (d) where the images show an increased density of nuclei in upper right quadrant of the image in comparison to the center of the image. 